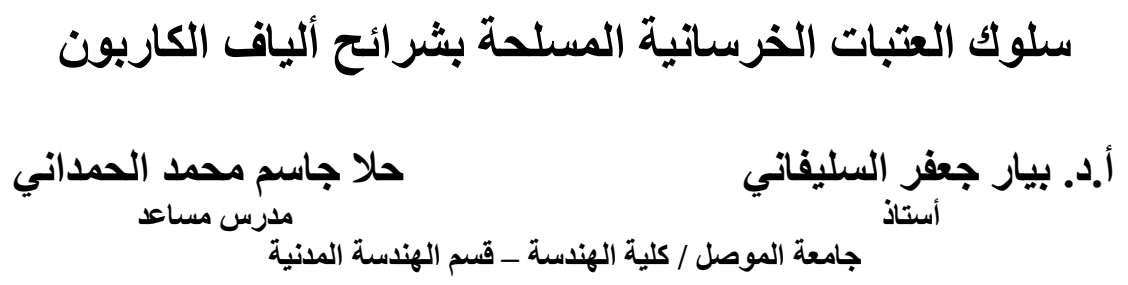

الخلاصة

تعددت الطرق والوسائل لزيادة قابلية تحمل المنشآت الخرسانية فقد استخدمت تقتية حديثة تمثلت باستخدام

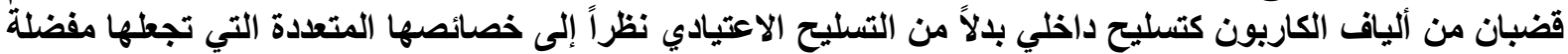

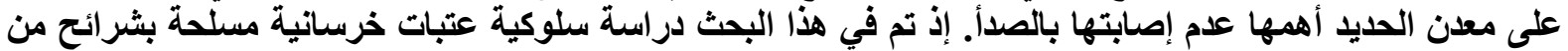

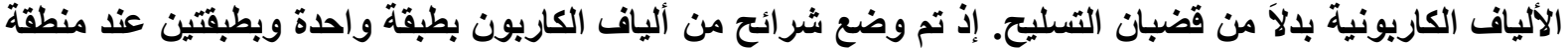

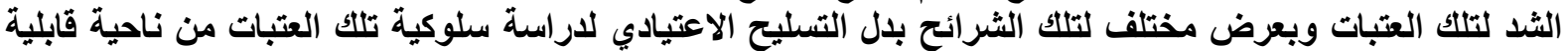

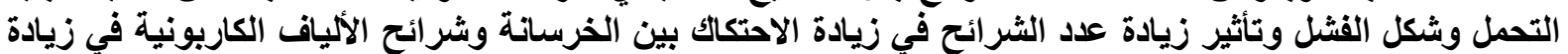

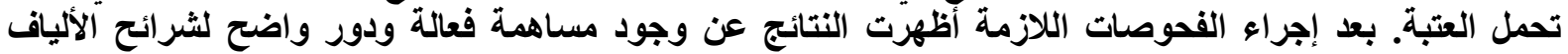

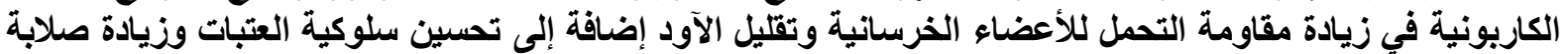

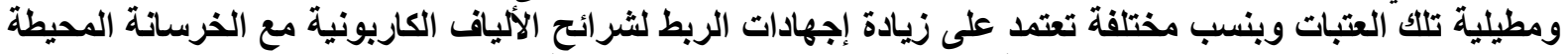

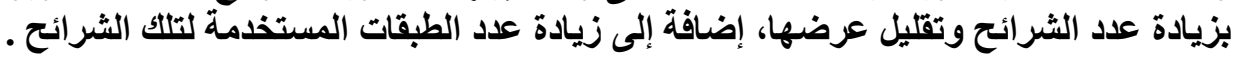

\title{
Behavior of Carbon Fiber Sheets Reinforced Concrete beam
}

\author{
Dr. Bayar J. Al-Sulayfani \\ Professor
}

\author{
Hala Jassim AL Hamdany \\ Assistant Lecturer
}

\begin{abstract}
One of the new technologies used in increasing the bearing capacity of concrete structures is the replacing of standard (normal) reinforcement by bars made of carbon fibers making use of its properties, which gives an advantage by being antirust. In this research, an experimental study of the behavior of concrete beams reinforced with strips of carbon fibers, (one or two layers), in the tension zone of the beams, with a variable width is performed to study the behavior of the beams from the point of view of their flexural capacity, failure mode, and the effect of the friction between the concrete and the fiber strip on load failure. After conducting the tests, the results revealed that an effective contribution and an obvious role of carbon strips fiber in improving the members bearing capacity and the overall behavior, also increasing the stiffness and ductility at different rates depending on the bond stress and number of strips.
\end{abstract}

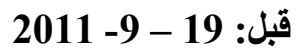

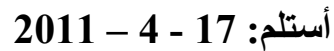




\section{المقدمة}

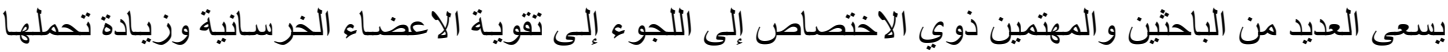

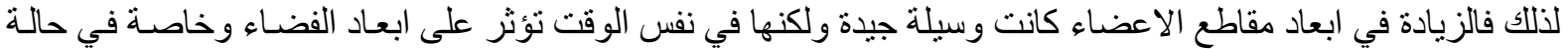

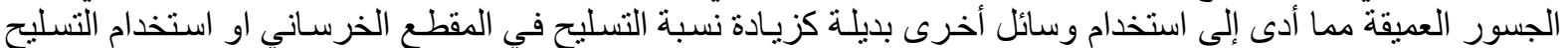

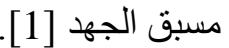

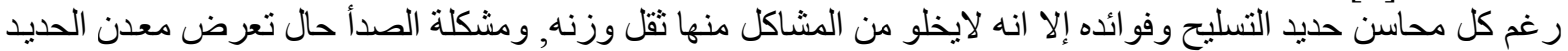

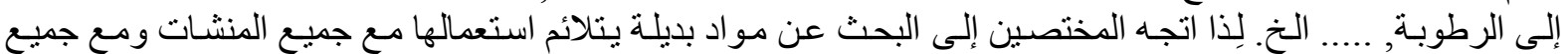

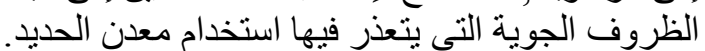

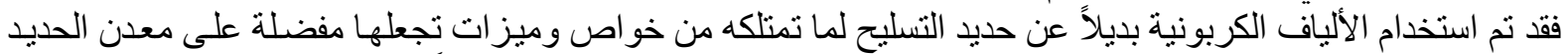

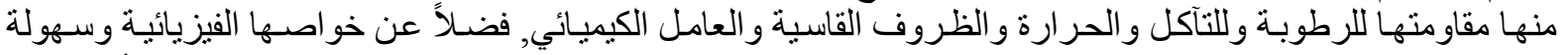

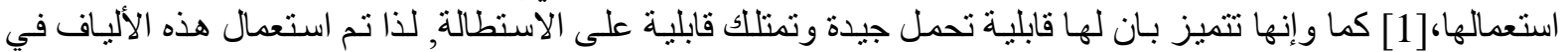

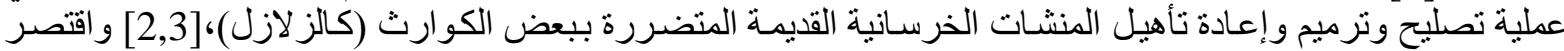

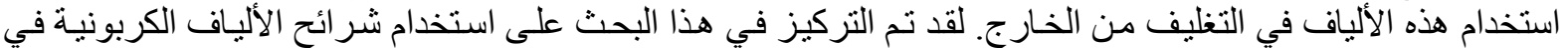

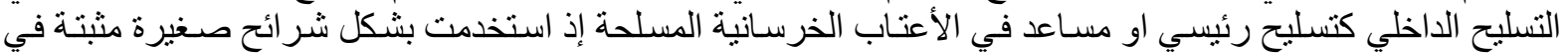
منطقة الثد بدلا من قضبان التسليح.

\section{الهزف من البحث}

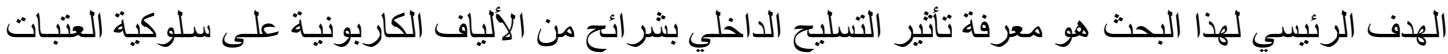

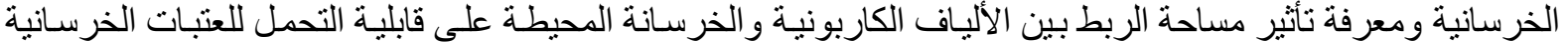
و عن اعتماد هذا التسليح في تحسين أداء هذه الأعضاء الإنشائية

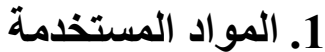

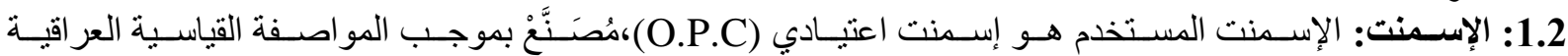

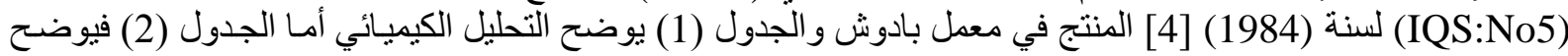
التحليل الفيزيائي للاسمنت المستخدم ومقارنتها مع حدود المو اصفة. 2.2: الماء: استُخدم ماء الثرب الاعنيادي الخالي من الثو ائب.

الجدول (1 ): يوضح التحليل الكيميائي للإسمنت المستخدم ومركباته الرئيسة [4]

\begin{tabular}{|c|c|c|}
\hline حدود المواصفة (IQS:5/1984)(2) & النسبة المئوية (\%) & اكاسيد عناصر الاسمنت \\
\hline $3-8$ & 5.6 & أوكسيد الألمنيوم \\
\hline $17-25$ & 21.52 & أوكسيد السليكا \\
\hline $0.5-6$ & 2.74 & أوكسيد الحديد \\
\hline $60-67$ & 62.55 & أوكسيد الكالسيوم CaO \\
\hline لا يزيد عن \% 2.8 & 2.54 & ثلاثي أوكسيد الكبريت SO \\
\hline لا يزيد عن 5\% & 3.23 & أوكسيد المغنيسيوم MgO \\
\hline \multicolumn{3}{|c|}{ مركبات الاسمنت الرئيسية } \\
\hline $31.03-41.05$ & 36.44 & ثلاثي سليكات الكالسيوم C \\
\hline $28.61-37.9$ & 34.20 & ثنائي سليكات الكالسيوم C \\
\hline $11.96-12.3$ & 12.07 & ثلاثي ألومينات الكالسيوم C \\
\hline $7.72-8.02$ & 7.98 & رباعي ألومينات الكالسيوم الحديدية \\
\hline
\end{tabular}


سلوك العتبات الخرسانية المسلحة بشرائح ألياف الكاربون

الجدول (2): يوضح التحليل الفيزيائي للإسمنت المستخدم [4]

\begin{tabular}{|c|c|c|}
\hline حدود المواصفة (IQS:5/1984)(\%) & نتيجة الفحص & الخصائص \\
\hline لا نزيد عن \% 10 & $\% 2$ & النعومة(نسبة المتبقي على منذل رقم 170) \\
\hline \multicolumn{3}{|c|}{ وقت التماسك } \\
\hline لا تقل عن 45 دقيقة & 140 & الابتدائي (دقيقة) \\
\hline لا تزيد عن 600 دقيقة & 385 & النهائي (دقيقة) \\
\hline \multicolumn{3}{|c|}{ مقاومة الانضغاط (MPa) } \\
\hline لا تقل عن ( 16 MPa) & 19.22 & أيام \\
\hline لا تقل عن (24 MPa) & 27.88 & 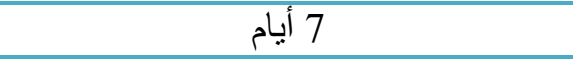 \\
\hline \multicolumn{3}{|c|}{ مقاومة الثد (MPa) مقد) } \\
\hline لا تقل عن(1.6 MPa) & 1.8 & 3 \\
\hline لا تقل عن (2.4 MPa) & 2.5 & 7 أيام \\
\hline
\end{tabular}

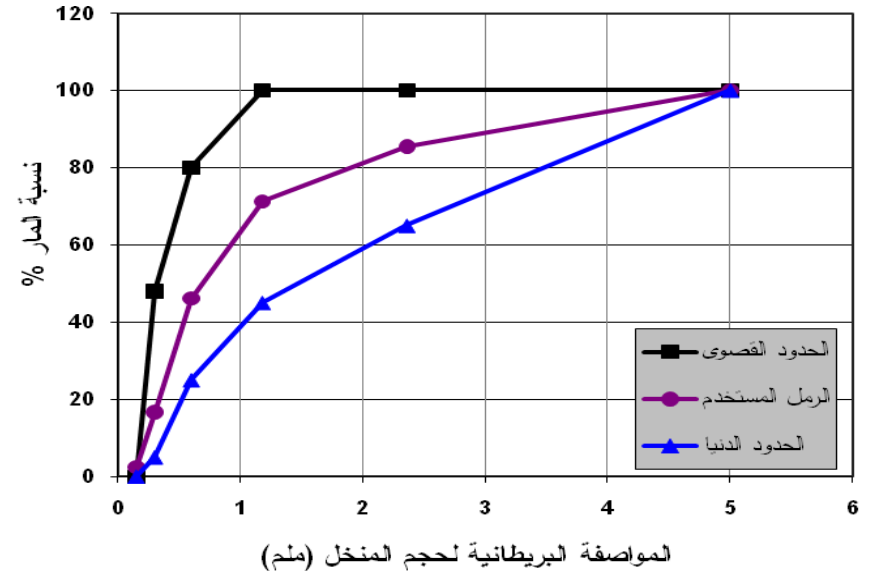

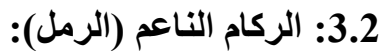

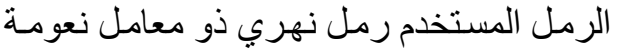

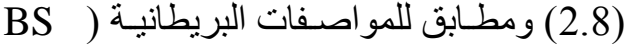

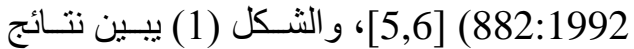
التحليل المنظلي للركام الناعم.

الثكل (1): يوضح التحليل المنظلي للركام الناعم المستخدم التيل المبن

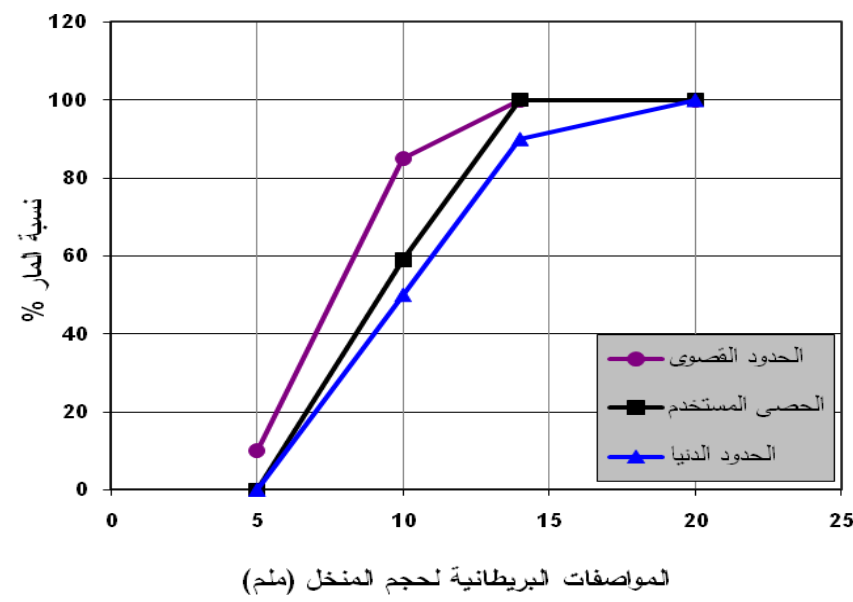

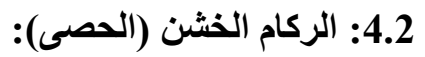
تم اسـتخدام الحصـى النهـري المحلـي المسدور

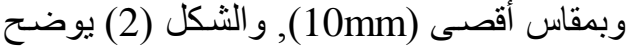
تدرج الحصى و المطابق للمو اصفات البريطانية

[5,6] (BS 882:1992)

$$
\text { الثكل(2): يوضح التحليل المنظلي للركام }
$$

5.2: ألياف الكاربون (CFRP) وخواصها

تم استخدام ألياف الكاربون من نوع (Sika Wrap Hex-230C) [7]. و الثكل (3) يوضـ شكل هذه الألياف،

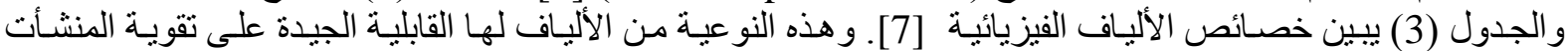

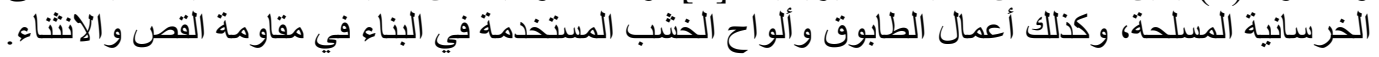




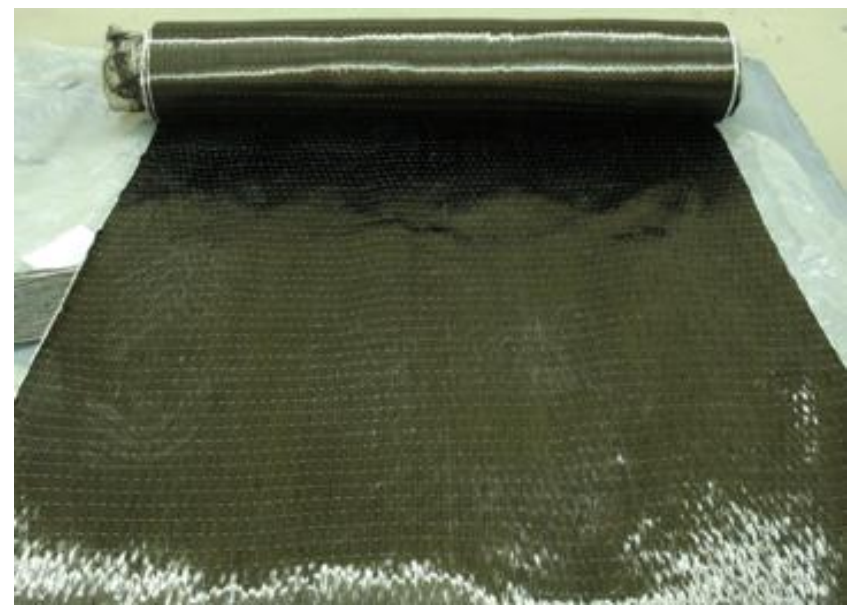

الثكل (3): يوضح شكل ألياف الكاربون (Sika Wrap Hex-230C)

$$
\text { الجدول (3): يوضح الخصائص الفيزيائية لألياف الكاربون }
$$

.[7](Sika Wrap Hex-230C)

\begin{tabular}{|c|c|c|}
\hline $1-$ & Fiber type & high strength carbon fibers \\
\hline $2-$ & Fiber orientation & $0^{\circ}$ ( unidirectional) \\
\hline $3-$ & Construction & $\begin{array}{l}\text { Warp: Carbon fibers } 99 \% \text { of total a real } \\
\text { weight) Weft: Thermoplastic heat-set } \\
\text { fiber }(1 \% \text { of total a real weight })\end{array}$ \\
\hline $4-$ & Areal weight & $10 \mathrm{gm} / \mathrm{m}^{2} \pm 220 \mathrm{gm} / \mathrm{m} 2$ \\
\hline $5-$ & Fiber density & $1.78 \mathrm{gm} / \mathrm{cm} 3$ \\
\hline $6-$ & Fabric design thickness & $0.12 \mathrm{~mm}($ Based on total carbon content $)$ \\
\hline $7-$ & Tensile strength of fiber & $4,100 \mathrm{~N} / \mathrm{mm}^{2}($ nominal $)$ \\
\hline $8-$ & Tensile E- modulus of fibers & $231.000 \mathrm{~N} / \mathrm{mm}^{2}$ (nominal) \\
\hline $9-$ & Strain at break of fibers & $1.7 \%($ nominal) \\
\hline $10-$ & Fabric length /roll & $300 / 600 \mathrm{~mm}$ \\
\hline $11-$ & Fabric width & 2 years from Date of production \\
\hline $12-$ & Shelf life & 1 roll in card board box \\
\hline $13-$ & Package &
\end{tabular}

3- الخلطة الخرسانية

تم اختيار المقاومة التصميمية للخلطة الخرسانية المستخدمة في صب العتبات الخرسانية بحدود (28MPa) عند التدات

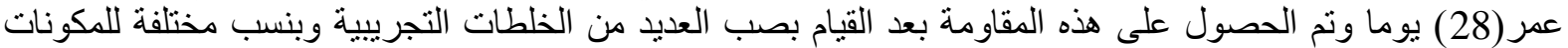

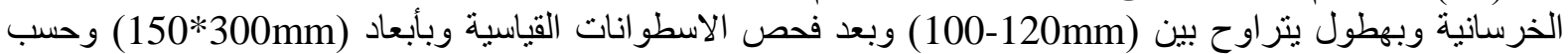

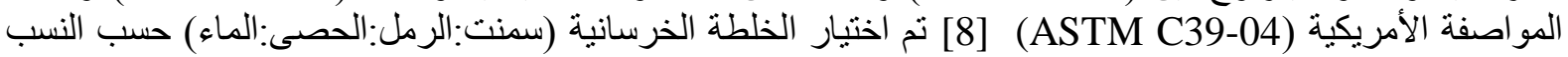

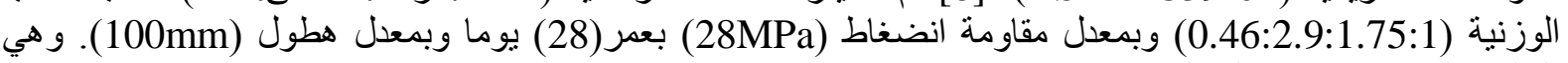
الخلطة المعتمدة في هذا البحث.

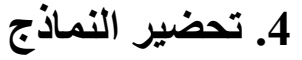

تم إعداد (10) أعتاب خرسانية بطول (1m) و عرض (150mm) وارتفاع (150mm)، اثنان منها غير مسلحةً

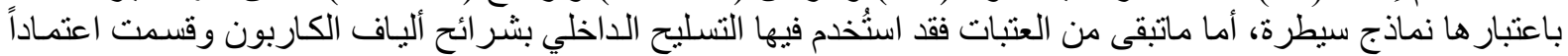
على عرض شر ائح ألياف الكاربون و عدد طبقاتها المستخدمة كما موضح في الثكل (4), إذ شملت الآتي: 
1- استخدمت (4) عتبات مسلحة بشريحتين من ألباف الكاربون بعرض (50mm) في كل طبقة كما موضح في الثكل (5-a,b)

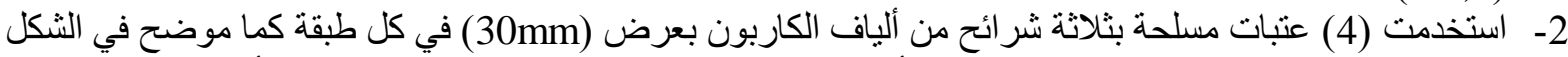
(5-a,c), اثنان منها مسلحة بطبقة واحدة من الألياف الكاربونية و اثثنان منها مسلحة بطبقتين من الألياف.

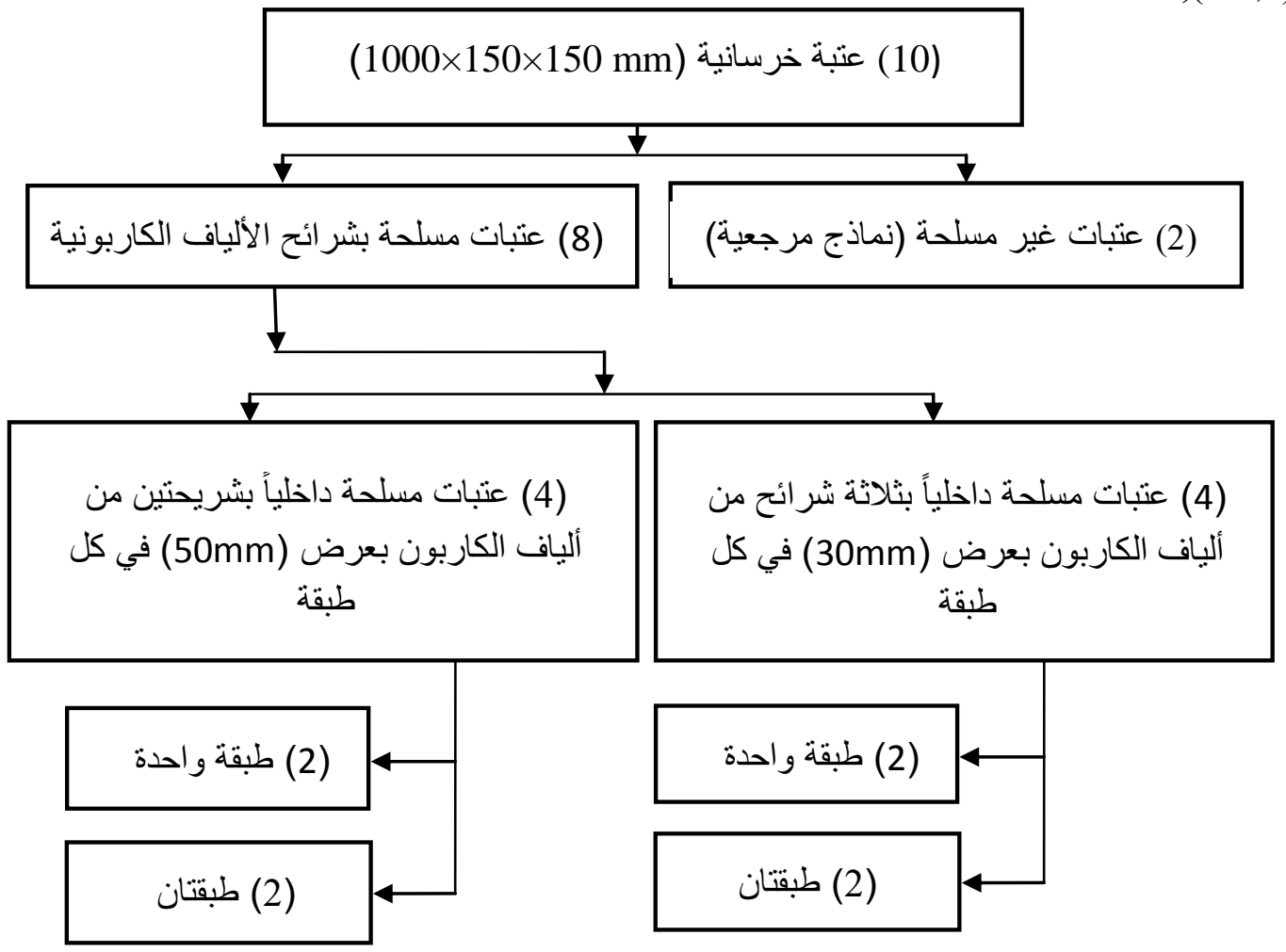

\section{الثكل (4): يوضح مخططاً للنماذج المستخدمة في البحث}

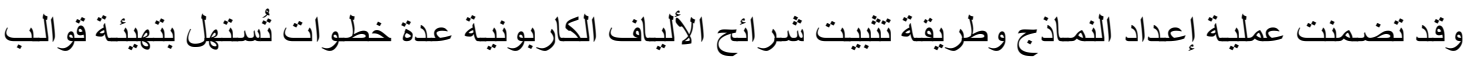

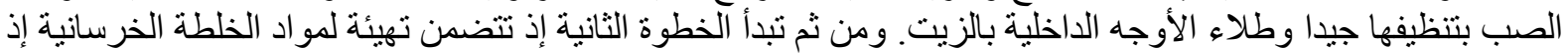

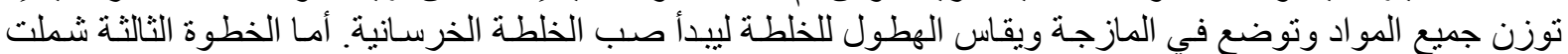

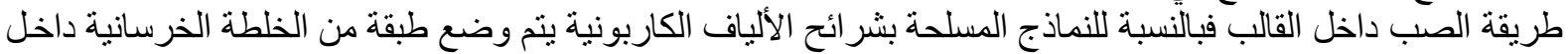

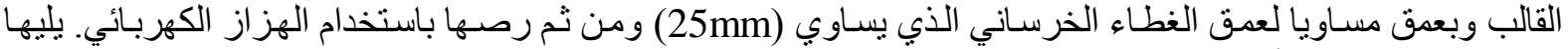

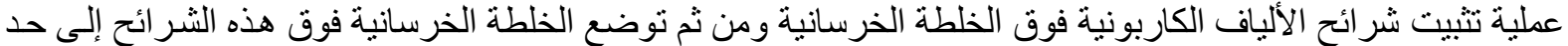

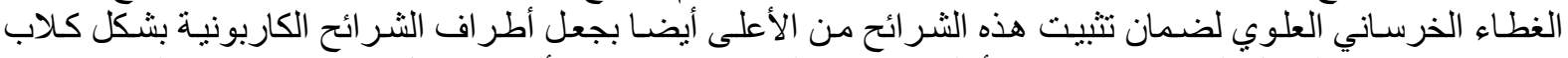
و (Hook) حصول فثل بالربط (Bonding) أي لتوفير طول التثبيت الكافي بحيث يضمن عدم حصول النئ النحاب للألافياف أثناء عملية

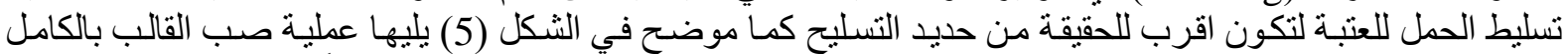

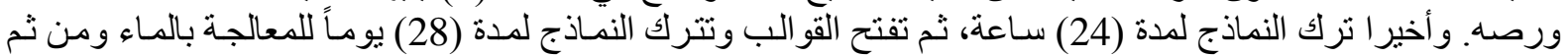

\section{5. الفحوصات والقياسـات المستخدمة في البحث}

استخدام جهاز فحص هيدروليكي (Universal Test Machine) ذو سعة قدرها (500KN) لإجر اء فحص نقطتين

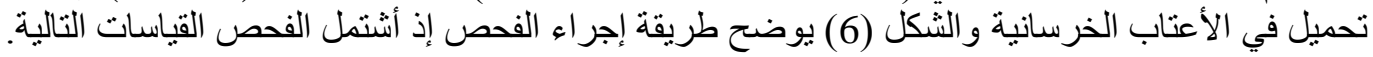

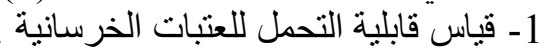

2- قياس الأود في وسط العتبة وذلك باستخدام مقياس قرصي مدرج (Dial gauge) بدقة (0.01mm). 


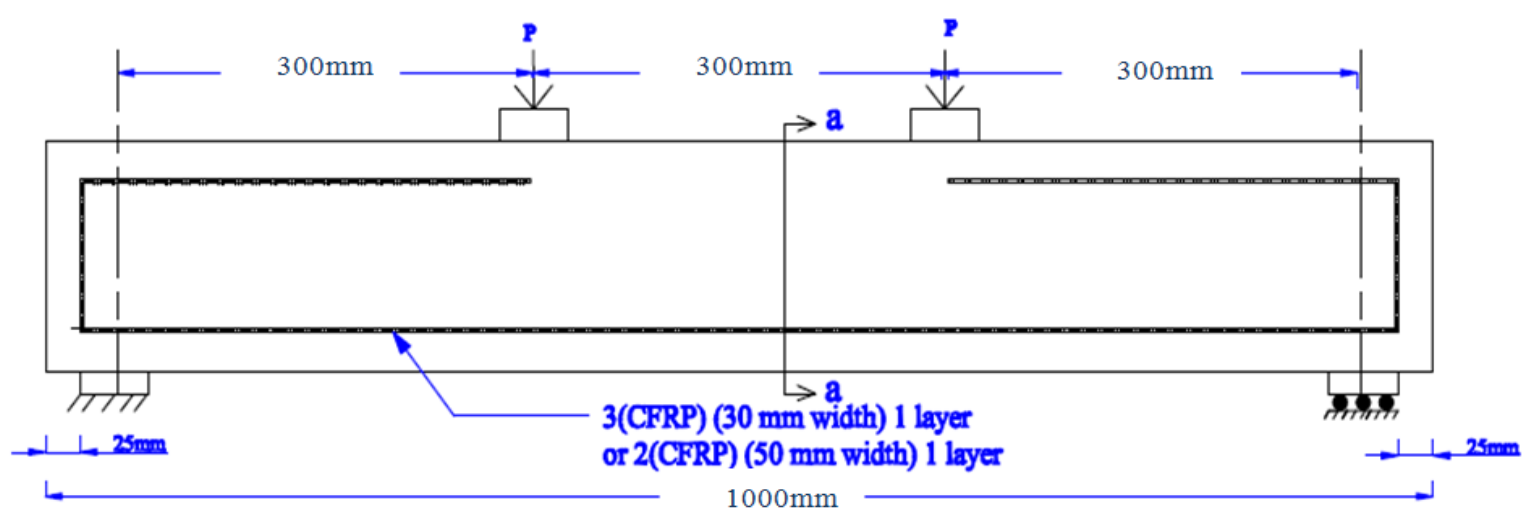

a): (a)
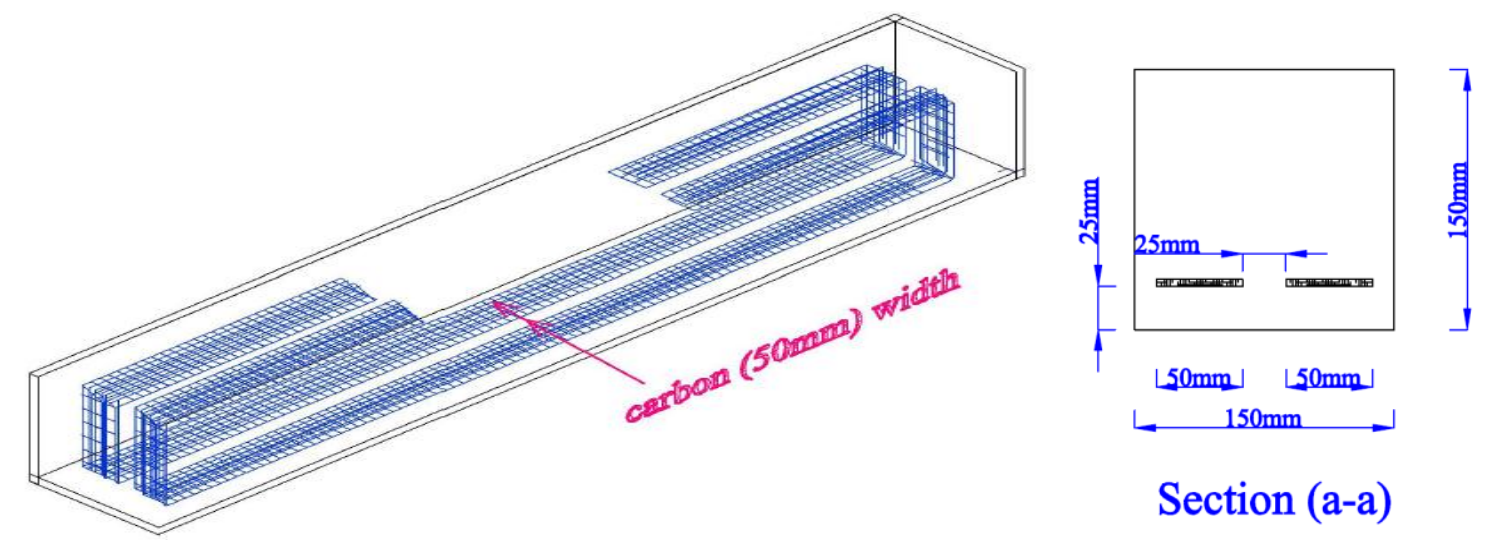

(b): يوضح هيكل التسليح لنموذج من العتبات الخرسانية المسلحة بثريحتين من ألياف الكاربون بعرض (50mm) في

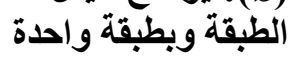
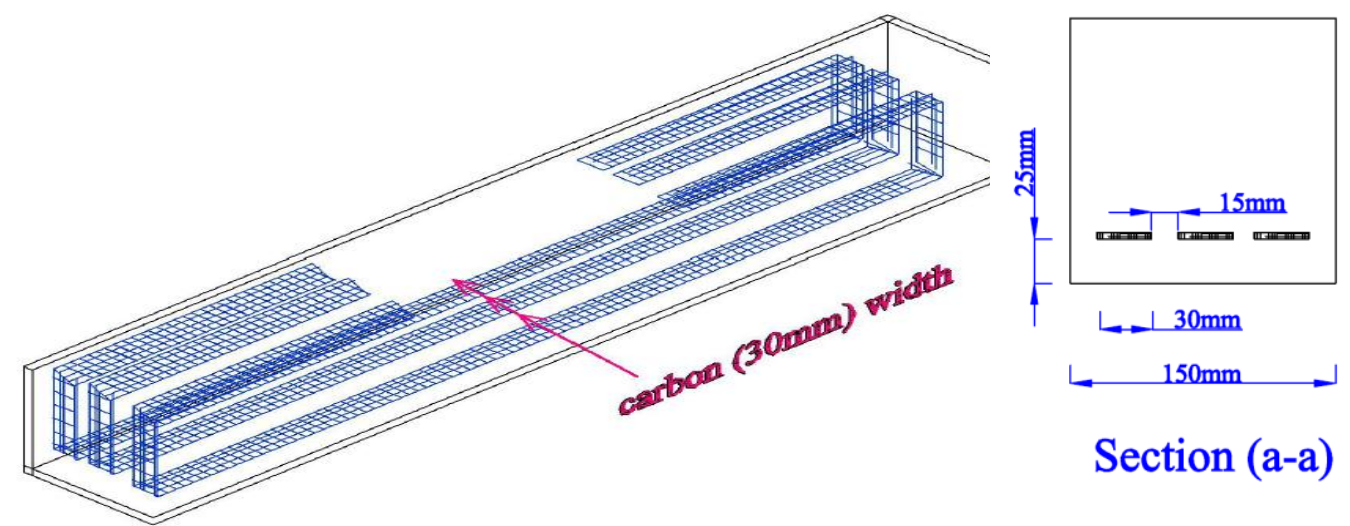

(c): يوضح هيكل التسليح لنموذج من العتبات الخرسانية المسلحة بثُلاثة شرائح من ألياف الكاريون بعرض (30mm) في الطبقة وبطبقة واحدة لخدة الثكل (5): أبعاد وشكل تسليح العتبة الخرسانية ونموذج لهيكل التسليح داخلياً بألياف الكاربون 


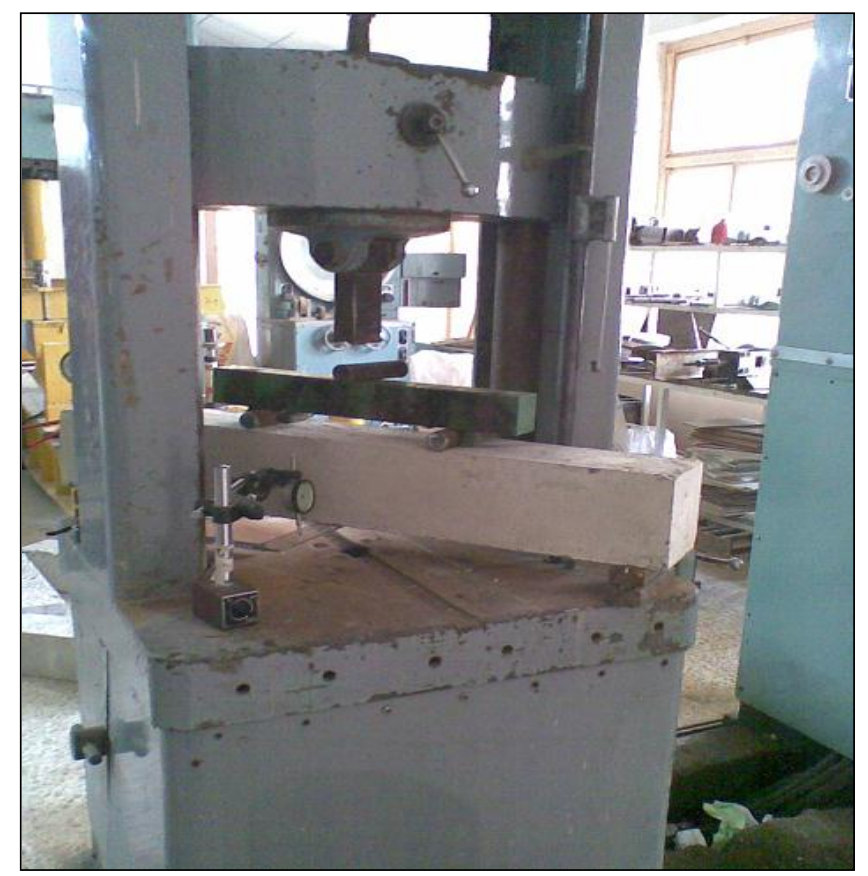

الشكل (6): يوضح جهاز فحص النماذج ونقاط التحميل في الأعتاب الخرسانية

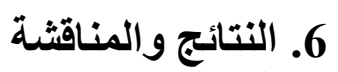

وشملت كل من:

1.6: دراسة تأثير التسليح بثرائح الألياف الكاربونية على حمل الفشل للعتبات الخرسانية

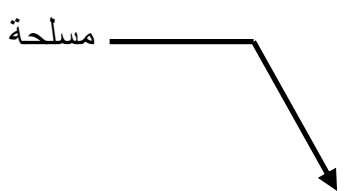

عدد طبقات الألياف الكاربونية

$$
\mathrm{BR}-(\text { or } 2)-(30 \mathrm{~mm} \text { or } 50 \mathrm{~mm})
$$
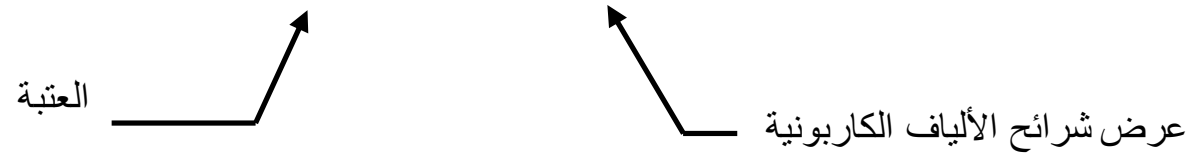

الثكل (7): يوضح ترميز للعتبات الخرسانية المستخدمة

الثـكل (7) يوضـح ترميـز للعتبـات الخرسـانية المسـتخدمة في هـذا البحـث. فحصـت جميـع العتبـات بجهـاز (Universal Test Machine)

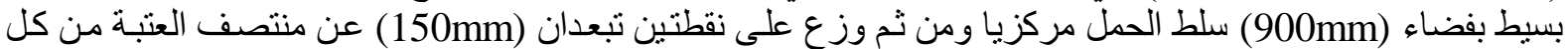

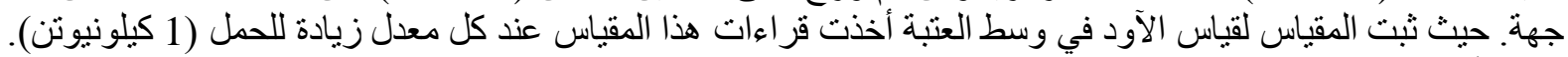

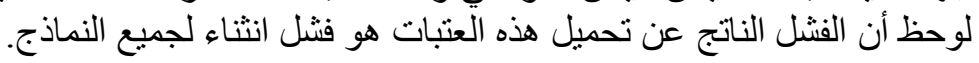

الجدول (4) يوضح نتائج الفحص إذ يبين نتائج أقصى حمل للعتبات عند الفشل والآود عند أقصى حمل كما يتبين من نتائج

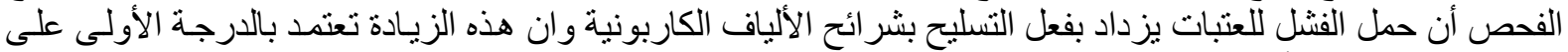

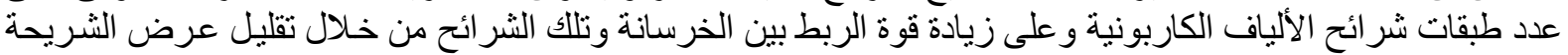

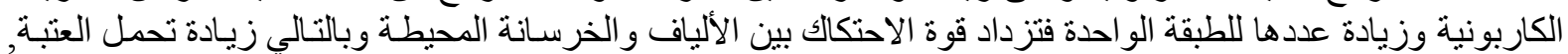


كما تظهر النتائج أن نسب الزيادة الحاصلة في حمل الفنل وما ينتج عنه من نقصان في الآود تبعاً للزيادة الحاصلة في عدد الطبقات و الزيادة في عدد شرائح الطبقة الواحدة.

الجدول (4): يوضح مقدار الفرق في الحمل الأقصى فضلاً عن الفرق الحاصل في قيمة الآود للعتبات الخرسانية

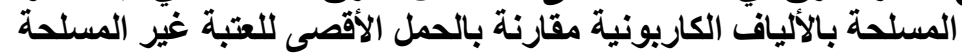

\begin{tabular}{|c|c|c|c|c|c|}
\hline النماذج & $\begin{array}{c}\Delta \text { الآود) } \\
\text { (ملم) }\end{array}$ & (كيلونيونن) & ف في حمل الفيل الزيادة & 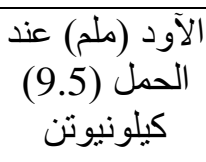 & \% الآود عنبة النقصان في الحمل \\
\hline No Reinforcement & 0.19 & 9.5 & ------- & 0.19 & ----- \\
\hline BR-1Layer-50mm & 2.5 & 15.0 & 57.8 & 0.14 & 25.2 \\
\hline BR-2Layer-50mm & 5.0 & 21.8 & 129.4 & 0.07 & 61.8 \\
\hline BR-1Layer-30mm & 2.3 & 19.0 & 100.0 & 0.07 & 63.1 \\
\hline BR-2Layer-30mm & 5.7 & 24.0 & 152.6 & 0.04 & 80.0 \\
\hline
\end{tabular}

الثكل (8) يوضح منحني علاقة (الحمل - الآود) للعتبات غير المسلحة و العتبات المسلحة داخليا بطبقة واحدة وطبقتين

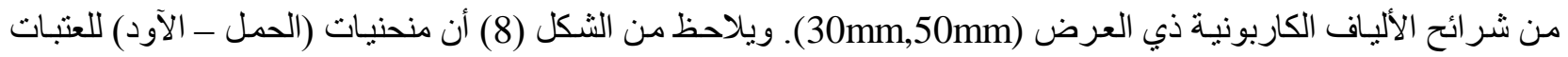

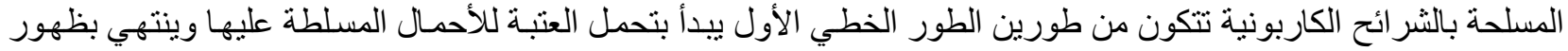

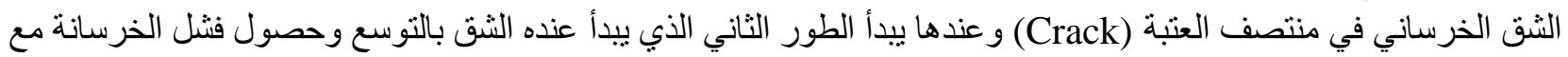

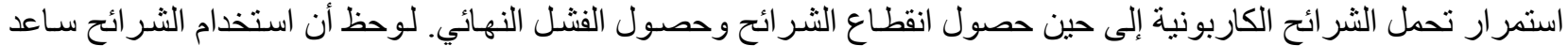

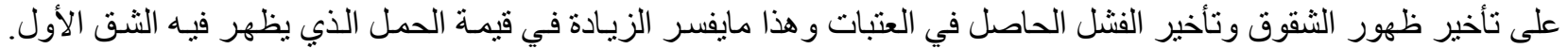

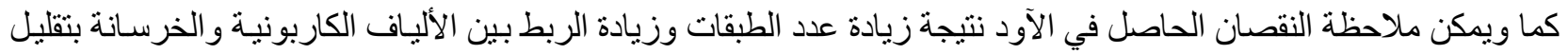

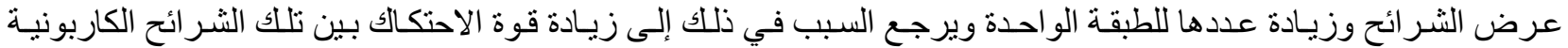

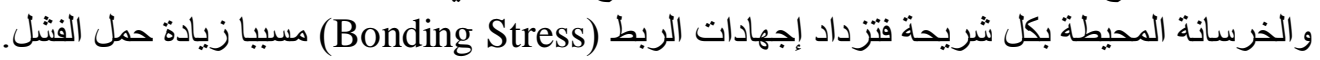

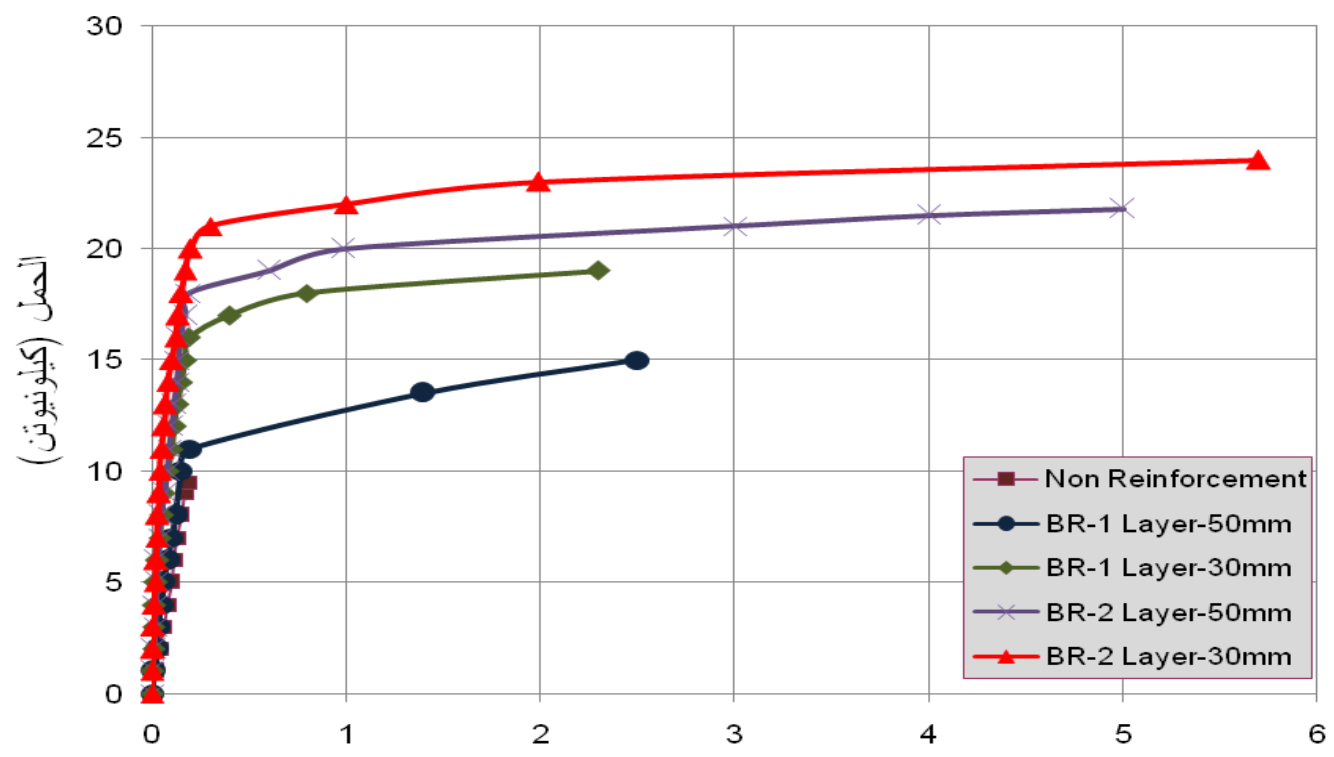

الآود منتصف العتبة (ملم) الآود) اللمقبات

الثكل (8): يوضح منحني علاقة (الحمل - الآود) للعتبات غير المسلحة و المسلحة داخليا بطبقة واحدة وطبقتين من شرائح الألياف الكاربونية ذي العرض (30mm,50mm) 
كما بمكن ملاحظة الزيادة الحاصلة في حمل الفشل و النقصـان الحاصل في الآود بزيـادة عدد طبقات الأليـاف

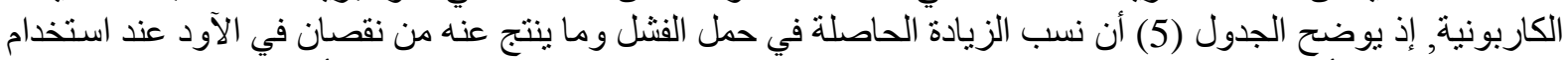
طبقة واحدة من الألياف الكاربونية كانت اكبر من الزيادة الحاصئلة عند استخدام طبقتين من تلاك الألياف.

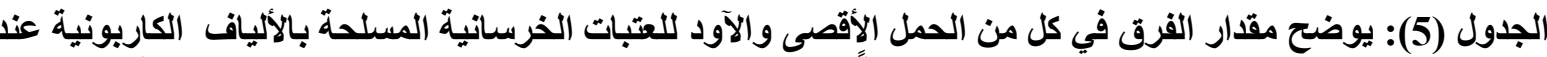

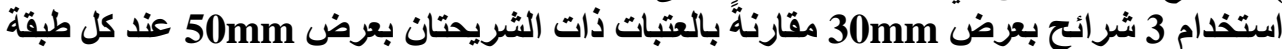

\begin{tabular}{|c|c|c|c|c|}
\hline النماذج & (كيلونيونن) حمل الفنل) & في حمل الفنل فيادة & الآود عند الحمل & ع عند الحمل (9.5) كيلونيون في الآود \\
\hline BR-1Layer-50mm & 15.0 & \multirow[t]{2}{*}{26.7} & 0.14 & \multirow[t]{2}{*}{50.7} \\
\hline BR-1Layer-30mm & 19.0 & & 0.07 & \\
\hline BR-2Layer-50mm & 21.8 & \multirow[t]{2}{*}{10.1} & 0.07 & \multirow[t]{2}{*}{47.6} \\
\hline BR-2Layer-30mm & 24.0 & & 0.04 & \\
\hline
\end{tabular}

\section{6: دراسة تأثير التسليح بشرائح الألياف الكاربونية على صلابة العتبات الخرسانية}

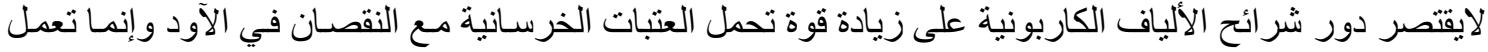

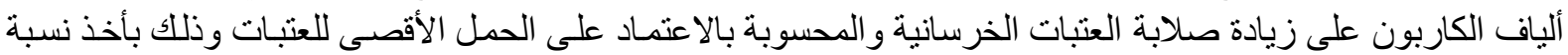

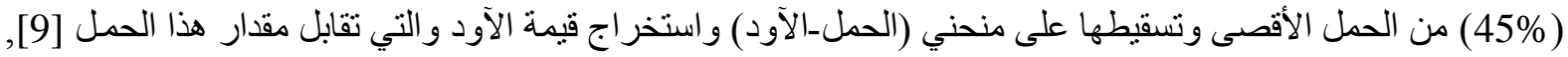

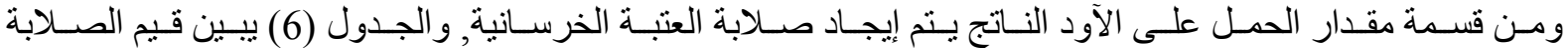
(k)(Stiffness)

\section{الجدول (6): يوضح قيم الصلابة للعتبات الخرسانية}

\begin{tabular}{|c|c|c|c|}
\hline النماذج & عدد الطبقات الفتل (كيلو نيوتن) & $\begin{array}{c}\text { (k) } \\
\text { (كيلونيوتن/ملم) }\end{array}$ \\
\hline No Reinforcement & 0 & 9.5 & 50.0 \\
\hline BR-1Layer-50mm & 1 & 15.0 & 71.1 \\
\hline BR-2Layer-50mm & 2 & 21.8 & 127.2 \\
\hline BR-1Layer-30mm & 1 & 19.0 & 225.0 \\
\hline BR-2Layer-30mm & 2 & 24.0 & \\
\hline
\end{tabular}

الجدول (6) يبين أن نسبة الزيادة الحاصلة في صلابة العتبات الخرسانية المسلحة داخليا بالألياف الكاربونية وبطبقة واحدة الكبة

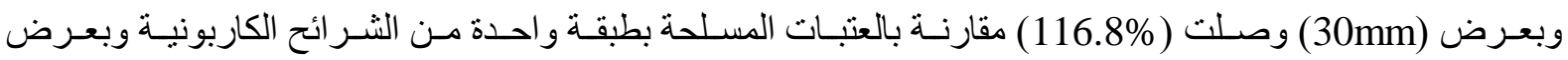

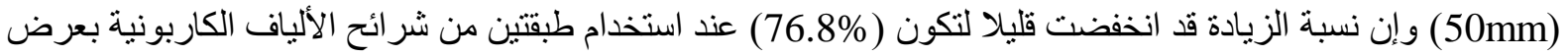

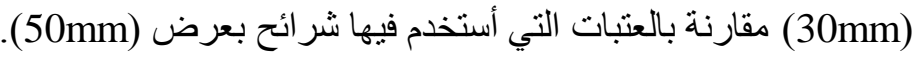

3.6: دراسة تأثير التسليح بثرائح الألياف الكاربونية على مطيلية ومتانة العتبات الخرسانية

يمكن ملاحظة الزيادة الحاصلة في المطيلية الحاصلة في العتبات الخرسـانية المسلحة بشر ائح الألياف الكاربونيـة

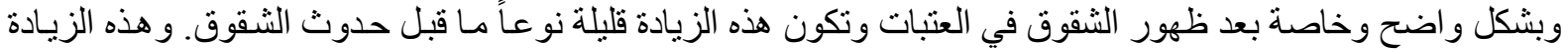
تزداد مع زيادة عدد طبقات الألياف الكاربونية. إذ يبين الجدول (7) المطيلية (Ductility) للعتبات الخرسانية الغير مسلحة

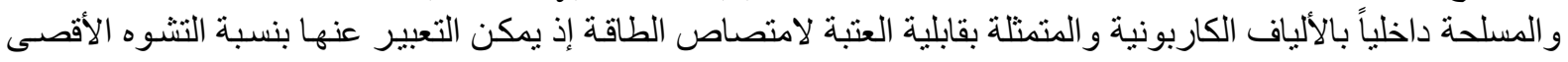
إلى التشوه في نقطة الخضوع (10) حساب المساحة تحت منحنيات (الحمل - الآود). 
الجدول (7): يوضح قيم المطيلية وطاقة الامتصاص للعتبات المستخدمة

\begin{tabular}{|c|c|c|c|c|}
\hline النماذج & الطبقات & (كيلونيونن) & 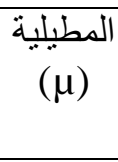 & 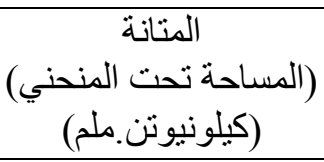 \\
\hline No Reinforcement & 0 & 9.5 & 0.0 & 1.0 \\
\hline BR-1Layer-50mm & 1 & 15.0 & 16.7 & 32.0 \\
\hline BR-2Layer-50mm & 2 & 21.8 & 35.7 & 100.7 \\
\hline BR-1Layer-30mm & 1 & 19.0 & 17.7 & 38.9 \\
\hline BR-2Layer-30mm & 2 & 24.0 & 57.0 & 129.1 \\
\hline
\end{tabular}

كما يبين الجدول أن نسب الزيادة الحاصلة في مطيلية ومتانة العتبات الخرسانية المسلحة داخليا بالألياف الكاربونية وبطبقة

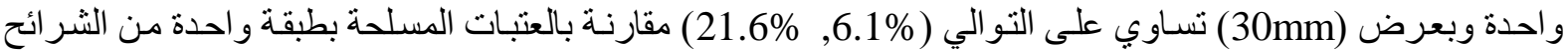
الكاربونية وبعرض (50mm) و إن هذه النسبة ازدادت عند استخدام طبقتنين من هذه الألياف لتكون (50) (59.64\%) 28.3\%) على التو الي. - ماني.

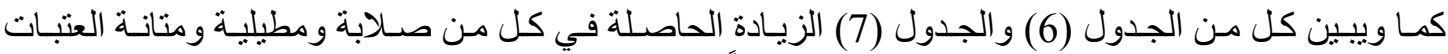

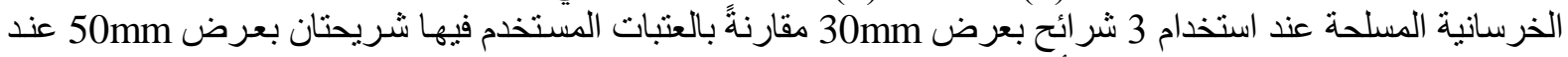

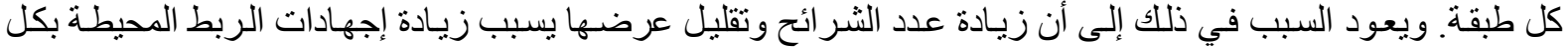

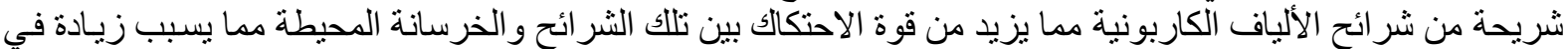
صلابة ومطيلية ومتانة العتبة الخرسانة و هذا بدوره يؤدي إلى زيادة الادئ حمل الفتل لتنلك العتبات. 4.6: دراسة تأثثر التسليح بشرائح الألياف الكاربونية على شكل الفشل للعتبات الخرسانية فنثل العتبات الخرسانية غير المسلحة كان فنشلاً قصفاً وفجائياً (Brittle and Sudden Failure) لايسبقه أب
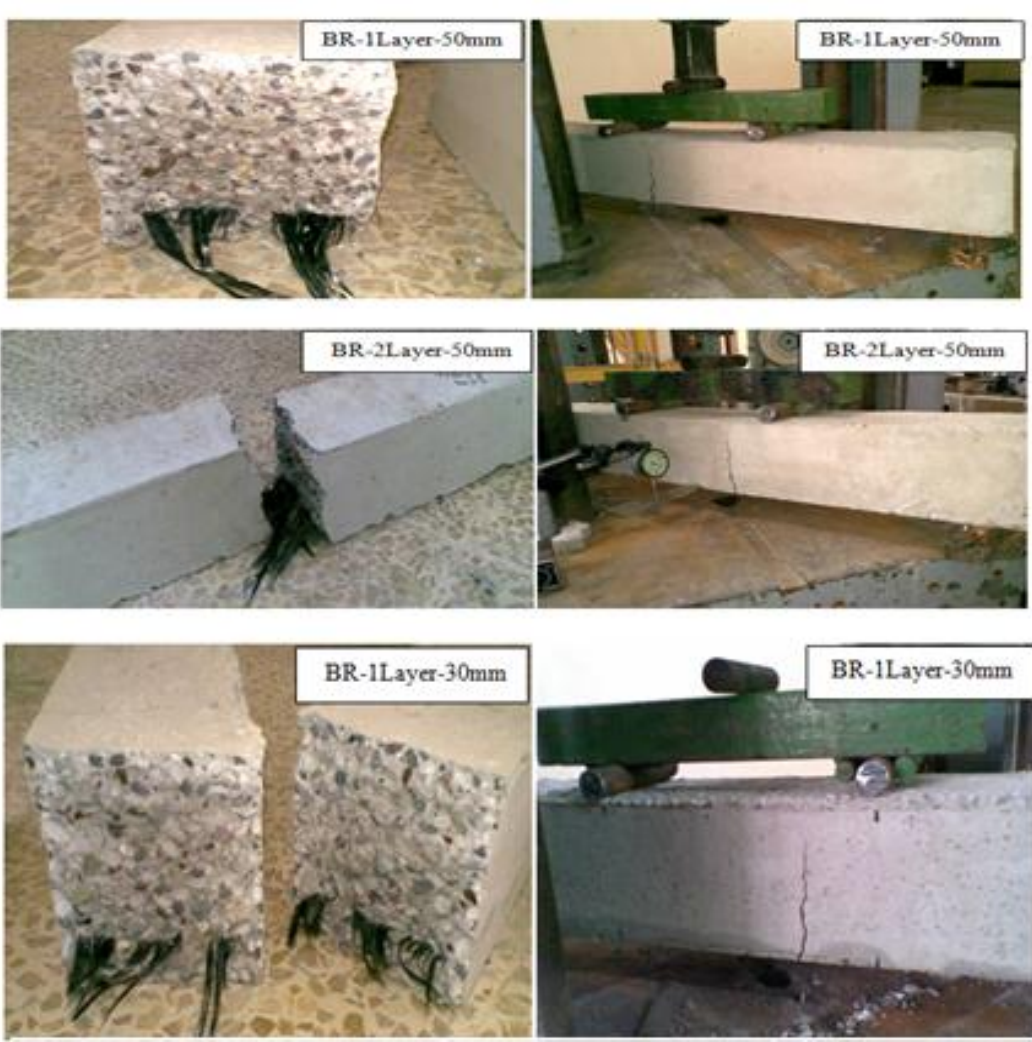

الثكل (9): يوضح شكل الفشل للعتبات الخرسانية المسلحة داخليا بألياف الكاربون

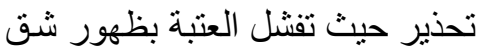

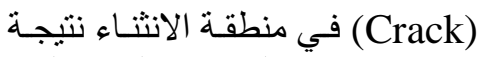

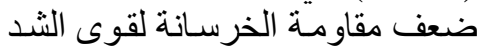
مقارنة بقوة الضغط إذ يمتد هذا الثقان

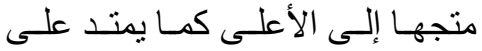

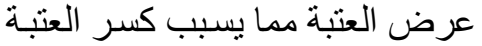

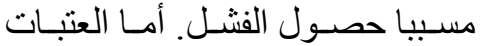
المسلحة بشـر ائح الألياف الكاربونيـة الفئة فقد كان الفشل مختلفاً إذ كان الفشـل تدريجياً مـع ظهور شق وسطي يبدأ بالاتسـاع بشكل تدريجي متجهيا إلى بـى

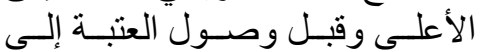

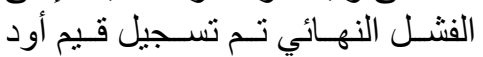
عالية بسبب حصول الفشل للخر سـانة

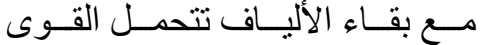

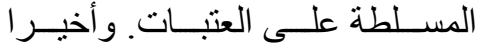

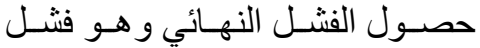
انثناء أيضا ولكن بشكل تدريجي إلى الى لـي

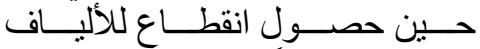
الكاربونية أيضاً بين نقطتي التحميل.

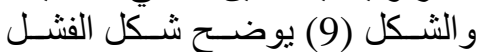
للعتبـات الخرسـانية المسـلحة داخليـاً بألياف الكاربون. 
من خلال الفحوصات المختبرية التي أجريت في هذه الدراسة، واستنادا على النتائج التي تم الحصول عليها خلصت

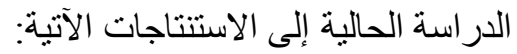

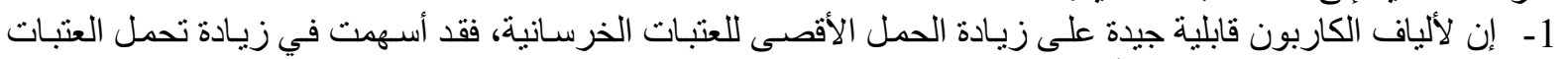

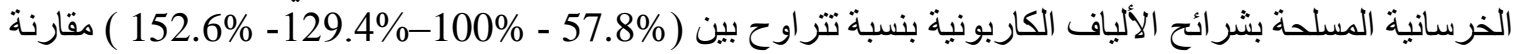

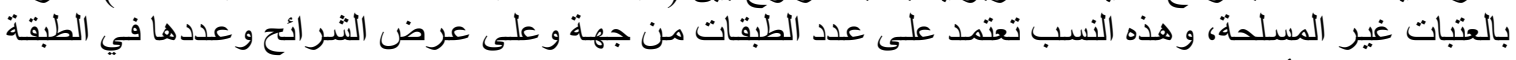

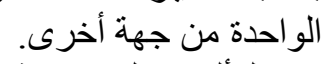

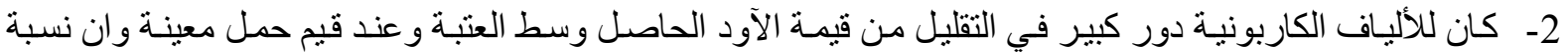

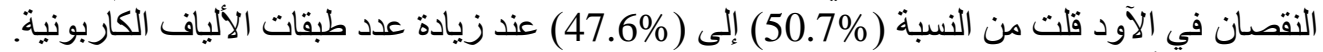

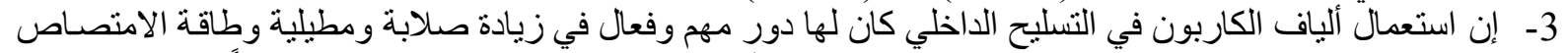

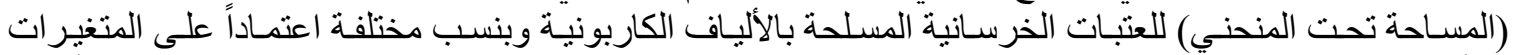

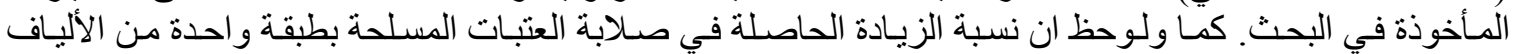

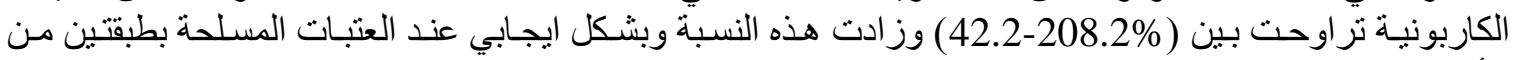
الألياف لتكون (154.4-350\%).

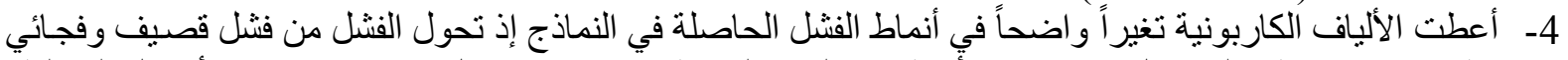

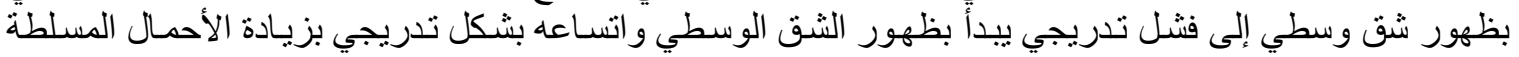

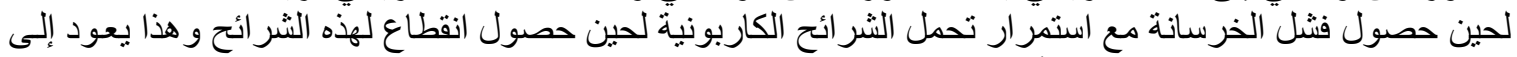
المطيلية العالية الني تمتاز بها هذه الألياف.

\section{References}

[1] Lamanna, A.J., "Flexural Strengthening of Reinforced Concrete Beams with Mechanically Fastened Fiber Reinforced Polymer Strips", Ph.D. Thesis, University of Wisconsin-Madison, 2002, 287pp

[2] Wong, R.Sh., "Towards Modelling of Reinforced Concrete Members with ExternallyBonded Fiber Reinforced Polymer (FRP) Composites", MSc. Thesis, University of Toronto, 2001, 320pp.

[3] Buell, T.W. and Saadatmanesh, H., "Strengthening Timber Bridge Beams Using Carbon Fiber", Journal of Structural Engineering, Vol. 131, No. 1, January, 2005, pp. 173-187.

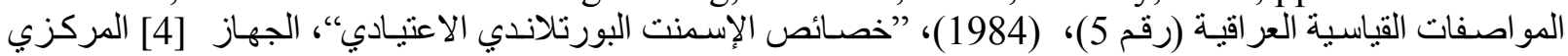

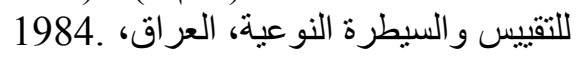

[5] (BS 882 : 1983), “Aggregates from Natural Sources for Concrete”, British Standards, Institute, London, 1983.

[6] (BS.882-1992), "Aggregates from Natural Source for Concrete", British Standard Institution, 1992.

[7] "Structural Strengthening With Sika Wrap Fabric System", Sika Services AGCH-8048 Zurich, Switzerland, p.415, www.Sika.com.

[8] ASTM C39-04, "Compressive Strength of Cylindrical Concrete Specimens", American Society for Testing and Materials.

[9] ACI committee 544.4R-88, "Design Considerations for Steel Fiber Reinforced Concrete", American Concrete Institute,1999, pp.1-18.

[10] Davies, P., Tanin, D.B. and Delpak, R., "Further Investigations in to the Ductility and Deformability of FRP Strengthened RC Elements", FRPRCS-8, University of Patras, Greece, Vol. 3, No. 11, July 2007, pp. 1-10.

$$
\text { تم اجراء البحث في كلية ألهنسة = جامعة ألموصل }
$$

